\title{
Mining Vavilov's Treasure Chest of Wheat Diversity for Adult Plant Resistance to Puccinia triticina
}

\begin{abstract}
Adnan Riaz, Queensland Alliance for Agriculture and Food Innovation, The University of Queensland, St. Lucia, QLD 4072, Australia; Naveenkumar Athiyannan and Sambasivam Periyannan, Queensland Alliance for Agriculture and Food Innovation, The University of Queensland; and Commonwealth Scientific and Industrial Research Organization (CSIRO) Agriculture, Canberra, ACT 2601, Australia; Olga Afanasenko, Department of Plant Resistance to Diseases of All Russian Research Institute for Plant Protection, Pushkin, 196608, Russia; Olga Mitrofanova, N. I. Vavilov Institute of Plant Genetic Resources, St. Petersburg, 190000, Russia; Elizabeth A. B. Aitken, School of Agriculture and Food Science, The University of Queensland; Evans Lagudah, CSIRO Agriculture; and Lee T. Hickey, Queensland Alliance for Agriculture and Food Innovation, The University of Queensland
\end{abstract}

\begin{abstract}
Leaf rust (LR) caused by Puccinia triticina, is among the most important diseases of wheat (Triticum aestivum L.) crops globally. Deployment of cultivars incorporating genetic resistance, such as adult plant resistance (APR) or all-stage resistance, is considered the most sustainable control method. APR is preferred for durability because it places lower selection pressure on the pathogen and is often polygenic. In the search for new sources of APR, here we explored a diversity panel sourced from the N. I. Vavilov Institute of Plant Genetic Resources. Based on DNA marker screening, 83 of the 300 lines were deemed to carry known APR genes; namely, $L r 34, L r 46$, and $\operatorname{Lr67}$. Interestingly, lines carrying $\operatorname{Lr67}$ were mostly landraces from India and Pakistan, reconfirming the likely origin of the gene. Rapid phenotypic screening using a method that integrates

assessment at both seedling and adult growth stages under accelerated growth conditions (i.e., constant light and controlled temperature) identified 50 lines carrying APR. Levels of APR corresponded well with phenotypes obtained in a field nursery inoculated using the same pathotype $\left(R^{2}=0.82\right)$. The second year of field testing, using a mixture of pathotypes with additional virulence for race-specific APR genes ( $L r 13$ and Lr37), identified a subset of 13 lines that consistently displayed high levels of APR across years and pathotypes. These lines provide useful sources of resistance for future research. A strategy combining rapid generation advance coupled with phenotyping under controlled conditions could accelerate introgression of these potentially novel alleles into adapted genetic backgrounds.
\end{abstract}

Bread wheat is considered the third most important food crop after maize and rice, providing a major source of carbohydrates and protein in the human diet (Ray et al. 2013). Along with the necessity to increase global wheat production to meet the needs of 9.7 billion people by 2050 (UN 2015), productivity is threatened by climate change (Asseng et al. 2015) and rapidly evolving diseases such as rusts caused by fungi from the genus Puccinia (Chaves et al. 2013). Among the rust diseases, leaf rust (LR) caused by Puccinia triticina Erikss. (previously known as $P$. recondita $\mathrm{f}$. sp. tritici) is an ongoing threat; yield losses due to its incidence have been reported in almost all wheat-growing regions, such as the United States, South America, Russia, Australia, China, India, South Africa, Mexico, Pakistan, Bangladesh, and Nepal. In these production environments, the annual yield loss due to LR ranges from 10 to $70 \%$, which varies greatly depending on the differences in crop growth stage, environmental conditions, and the degree of plant defense (Huerta-Espino et al. 2011; Niks et al. 2015).

Cultural measures such as removal of the "green bridge" (i.e., off-season wheat cultivation), cultivation of early-maturing varieties, and use of fungicides can minimize losses due to LR; however, they have limitations (Jørgensen et al. 2014). The most effective method is deployment of genetic resistance which, if managed correctly, can be sustainable.

Adult plant resistance (APR) is best expressed at the adult plant stage and is often underpinned by multiple genes, each quantitatively contributing a minor effect to the plant defense level (Ellis et al. 2014;

Corresponding author: L. Hickey; E-mail: 1.hickey@uq.edu.au

*The $\boldsymbol{e}$-Xtra logo stands for "electronic extra" and indicates that one supplementary table is published online.

Accepted for publication 22 September 2016.

C 2017 The American Phytopathological Society
Lagudah 2011; Niks et al. 2015). APR is underpinned by genes that influence factors such as latent period, pustule size, and infection frequency in order to provide a "slow rusting" or partial resistance phenotype (Caldwell 1968; Ellis et al. 2014; McIntosh et al. 1995; Niks et al. 2015; Spielmeyer et al. 2013). Therefore, APR is considered more durable than all-stage resistance (ASR) or seedling resistance, which is typically governed by a major gene providing a hypersensitive response (HR). Although APR is often nonrace specific, there are exceptions, where some genes provide racespecific resistance (such as Lr13) (Ellis et al. 2014) and confer an HR (such as Lr48) (Bansal et al. 2008). When APR genes are combined, they often act additively, and high levels of resistance (or near-immunity) can be achieved (Singh et al. 2014). The cloning of Lr34 (Krattinger et al. 2009) and Lr67 (Moore et al. 2015) has provided perfect markers for marker-assisted selection and facilitates deployment of these genes in cultivars. However, if these genes are deployed alone in cultivars, it could make them more vulnerable to pathogen evolution. This highlights the importance of searching for new or additional sources of resistance for creating gene stacks or pyramids which, if deployed in this form, will prolong the life of these valuable genes.

There are approximately 850,000 viable wheat accessions stored in seed banks worldwide (Mitrofanova 2012). Although this represents a huge array of genetic diversity, identifying accessions carrying novel sources of rust resistance is challenging. Traditionally, APR is determined by phenotyping seedlings in the glasshouse and adult plants in the field. However, the success of field-based phenotyping is dependent on weather conditions favorable for epidemic development (Hickey et al. 2012) and restricted to local pathotypes. Moreover, screening in the field is often limited to just once a year in the wheat growing season. Using this approach to evaluate large numbers of seed bank accessions is a slow process. A new method reported by Riaz et al. (2016) permits rapid phenotyping for APR to LR in wheat grown under controlled environmental conditions. The method exploits constant light and controlled temperature to rapidly obtain adult plants. The technique involves two sequential 
inoculations, one at the seedling stage and another at the adult stage, to phenotype APR within 7 weeks and can be performed all year round.

Here, we search for new sources of APR to LR by mining a diverse panel of 300 wheat accessions sourced from the N. I. Vavilov Institute of Plant Genetic Resources (VIR) in St. Petersburg, Russia. The institute was originally formed in 1901 and was later named after the great Russian botanist N. I. Vavilov, best known for his theory relating to "the centers of origin of cultivated plants". Vavilov and his colleagues led various expeditions to different parts of the world and collected a huge diversity of wheat (Riaz et al. in press). Currently, the VIR wheat collection contains 38,430 samples, of which 29,209 are bread wheat (i.e., Triticum aestivum L.), 6,199 are durum wheat (i.e., T. durum Desf.) and 3,022 are wild wheat (Mitrofanova 2012). In the present study, we applied DNA markers to screen for known APR genes (i.e., $\operatorname{Lr} 34, \operatorname{Lr} 46$, and $\operatorname{Lr} 67$ ) and performed rapid phenotyping under controlled conditions. We investigated the distribution of known APR genes in the diversity panel and provide insight on their likely origin. Based on initial screening, we evaluated a promising subset in the field over 2 years and identified valuable genetic materials for future research aiming to characterize new APR genes, which are required to diversify resistance factors in breeding programs.

\section{Materials and Methods}

Plant materials. This study examined a diverse panel of wheat accessions comprising 300 single-seed descent (SSD) lines (295 hexaploid and 5 tetraploid) sourced originally from VIR, St. Petersburg, Russia. The 295 hexaploid SSD lines were previously characterized for genetic diversity and population structure using the genotyping-by-sequencing Diversity Arrays Technology platform by Riaz et al. (in press). For line purification, a single plant for each of the 300 VIR accessions was grown in the glasshouse and subjected to a generation of SSD to develop genetically stable lines for subsequent genotypic and phenotypic analyses. The derived SSD lines were assigned new Australian Grain Genebank (AGG) accession numbers (Supplementary Table S1). The panel includes landraces $(n=136)$, cultivars $(n=36)$, breeding lines $(n=10)$, and lines with unknown cultivation status $(n=118)$. The pure seed for SSD lines was used in all experiments conducted in this study. A set of disease standards were also included: 'Thatcher', 'Avocet', and near-isogenic lines in the Avocet background (i.e., Avocet $+L r 34$ and Avocet+Lr46).

Pathogen materials. Two LR pathotypes were used in this study: pathotype 104-1,2,3,(6),(7),11,13 and pathotype 76-1,3,5,7,9,10,12,13+ Lr37 (Table 1). These pathotypes are prevalent in eastern and western wheat-growing regions of Australia (Park and Bariana 2013; Park and Wellings 2011; Park et al. 2002, 2015). The rust cultures used in this study were maintained through single-spore culture technique using susceptible 'Morocco' wheat.

Polymerase chain reaction marker screening for known APR genes. Three hundred SSD lines in the diversity panel were screened with polymerase chain reaction (PCR)-based markers for previously reported LR APR genes; namely Lr34, Lr46, and Lr67. Genomic DNA for each line was extracted using the method reported by Diversity Array Technology Pty. Ltd. (http://www.triticarte.com. au). The screening of $\operatorname{Lr} 34$ was performed using the gene-specific cleaved amplified polymorphic sequence (CAPS) marker cssfr 5 (forward primer Lr34SPF and reverse primer L34DINT13R2), as described by Lagudah et al. (2009). The cssfr 5 marker enables accurate identification of the gene in diverse wheat germplasm (Lagudah et al. 2009). For detection of $L r 46$, the CAPS marker named csLV46 was used (unpublished data). For detection of Lr67, a gene-specific single-nucleotide polymorphic marker (i.e., SNP1-TM4) was used (Moore et al. 2015).

Rapid phenotyping for seedling and APR. The integrated seedling and adult plant method developed by Riaz et al. (2016) was employed to phenotype the 300 SSD lines at The University of Queensland, St. Lucia, Queensland, Australia. To encourage synchronous germination for all genotypes, seed were imbibed with water for $24 \mathrm{~h}$ at room temperature and were placed in a refrigerator $\left(4^{\circ} \mathrm{C}\right)$ for $48 \mathrm{~h}$. Germinated seed were transplanted into 140-mm ANOVApot pots (http:// www.anovapot.com/index.php) filled with a potting medium consisting of $70 \%$ composted pine bark fines ( 0 to $5 \mathrm{~mm}$ ) and $30 \%$ coco peat with a $\mathrm{pH}$ of 5.5 to 6.5. Slow-release Osmocote fertilizer was applied at a rate of $2 \mathrm{~g} /$ pot. Three seeds of each line were clumped together at one position, where each pot had four positions. Plants were grown under regular glasshouse conditions at temperatures of $22^{\circ} \mathrm{C}$ (day) and $17^{\circ} \mathrm{C}$ (night) and a diurnal photoperiod $(12 \mathrm{~h})$. After 10 days, seedlings were inoculated with pathotype 104-1,2,3,(6),(7),11,13, where urediniospores were suspended in light mineral oil (Isopar 6) at a concentration of $6 \times 10^{5}$ spores $/ \mathrm{ml}(0.005 \mathrm{~g} / \mathrm{ml})$ and applied using an airbrush (IWATA power jet lite). At 12 days postinoculation, seedlings were assessed for infection type (IT) using the 0-to-4 Stakman scale (Stakman et al. 1962). Lines that displayed an IT of $<3$ were considered resistant (R).

Following the seedling assessment, plants were grown under accelerated growth conditions (AGC), which were achieved by adopting constant $(24 \mathrm{~h})$ light and a 12-h cycling temperature regime of 22 and $17^{\circ} \mathrm{C}$ (Riaz et al. 2016). The plants were grown for 2 weeks under AGC and were reinoculated with a suspension of LR urediniospores (i.e., pathotype 104-1,2,3,(6),(7),11,13), as described above. Inoculation of plants after this period of growth provided phenotypes similar to adult plants in the field (Riaz et al. 2016). At 12 days postinoculation, the LR response was recorded for the flag-2 leaf using the 0-to-4 Stakman scale, where IT $<3$ was considered $\mathrm{R}$.

Field evaluation. SSD lines displaying APR under AGC that lacked known APR genes (based on marker screening) were evaluated for resistance in the field over two consecutive years (from July to October 2014 and 2015) at Redlands Research Facility, Queensland, Australia. The LR-susceptible (S) genotype Morocco was used as a disease spreader, where two rows of Morocco were sown between each bay comprising two rows of hill plots. Lines were sown as nonreplicated hill plots. A set of disease standards, including Thatcher, Avocet, Avocet $+L r 34$, and Avocet $+L r 46$, were replicated throughout the nursery to monitor the LR epidemic progression. The LR epidemic was initiated by transplanting rust-infected Morocco seedlings into the field among the spreader rows about 5 weeks after sowing. Favorable conditions for the disease were maintained by applying sprinkler irrigation in the late evenings. Plants were assessed when the rust epidemic had sufficiently developed on disease standards to allow a clear differentiation between $\mathrm{S}$ and $\mathrm{R}$ genotypes.

In 2014, the LR nursery was inoculated with LR pathotype 104$1,2,3,(6),(7), 11,13$ - the same pathotype used for screening under controlled conditions. The disease response was assessed on a wholeplot basis using the modified Cobb scale (Peterson et al. 1948). Multiple disease assessments were conducted from late tillering to early grain

Table 1. Virulence profile of Puccinia triticina pathotypes used in this study

\begin{tabular}{|c|c|c|}
\hline Pathotype & Virulent on genes ${ }^{\mathrm{a}}$ & Avirulent on genes \\
\hline $104-1,2,3,(6),(7), 11,13^{\mathrm{b}}$ & Lr1, Lr3a, Lr14a, Lr16, Lr17a*, Lr20, Lr24, Lr27+31* & Lr2a, Lr3ka, Lr13, Lr15, Lr17b, Lr23, Lr26, Lr28, Lr37 \\
\hline $76-1,3,5,7,9,10,12,13+\operatorname{Lr} 37^{\mathrm{c}}$ & $\begin{array}{l}\text { Lr3a, Lr3ka, Lr13, Lr14a, Lr17a, Lr17b, Lr20, } \\
\quad \text { Lr24, Lr26, Lr37 }\end{array}$ & $L r 1, L r 2 a, L r 15, L r 16, L r 23, L r 27+31 \operatorname{Lr} 28$ \\
\hline
\end{tabular}

\footnotetext{
${ }^{a}$ Asterisk indicates that the pathotype is partially virulent on the gene.

b Single pathotype used in rapid phenotyping seedling and APR under accelerated growth conditions and the 2014 field experiments.

${ }^{\mathrm{c}}$ The additional pathotype used in the 2015 field experiment.
} 
filling (i.e., 70, 77, and 86 days after sowing [DAS]). Host response and disease severity data were used to calculate the coefficient of infection, as per Loegering (1959). Lines that displayed an LR response between $\mathrm{R}$ to moderately resistant-moderately susceptible (MRMS) were considered resistant.

In 2015, the LR field nursery was inoculated with a mixture of two pathotypes: 104-1,2,3,(6),(7),11,13 and 76-1,3,5,7,9,10,12,13+Lr37. Notably, in comparison with the 2014 screening, this provided additional virulence for race-specific APR genes Lr13 and Lr37. Plants were assessed using the 1-to- 9 scale reported by Bariana et al. (2007). Two disease assessments (i.e., 78 and 85 DAS) were conducted from late tillering to early grain filling. Lines that displayed an LR response $\leq 5$ (i.e., MRMS) were considered resistant.

Statistical analysis. In the integrated seedling and adult plant experiment under AGC, disease response was evaluated using the 0-to-4 Stakman scale, which contains both numbers (e.g., 0, 1..4) and symbols (e.g., ;, +, and others). The symbols represent variations in the LR response, which were indicated by the use of "-" (i.e., lower than average for the class) and " + " (i.e., higher than average for the class), as well as " $c$ " and " $n$ " to indicate more than usual degrees of chlorosis and necrosis, respectively. The data were converted to a 0-to-9 scale, where $0=$ immune and $9=$ very susceptible according to Riaz et al. (2016). The converted IT were as follows: $0 ;$; ;n, ; 1-, 1, 1+, 2-, 2, 2+, $2++, 3-, 3,3+, 3++$, and 4 were coded as $0,0.5,1,2.5,3,3.5,4,5$, $5.5,6,6.5,7,8,8.5$, and 9 , respectively. For heterogeneous IT, where no direct conversion value was available, each score was converted individually to the 0 -to- 9 scale and the average calculated. The converted datasets were used to generate frequency distributions and illustrate resistance levels for lines carrying known APR genes.

In order to compare disease response of the lines potentially carrying new sources of APR across the four experiments (i.e., seedling, AGC, and 2 years of field assessment) principal component analysis was performed and results visualized in the form of a biplot using GenStat (17.1 2000-2015; VSN International Ltd.) (GenStat.co.uk).

\section{Results}

Geographical distribution of known APR genes. In total, 83 lines in the diversity panel were deemed to carry known genes for APR. The APR genes $L r 34, L r 46$, and $\operatorname{Lr} 67$ were present in 9, 13, and 48 lines with known origin information, respectively (Fig. 1A,
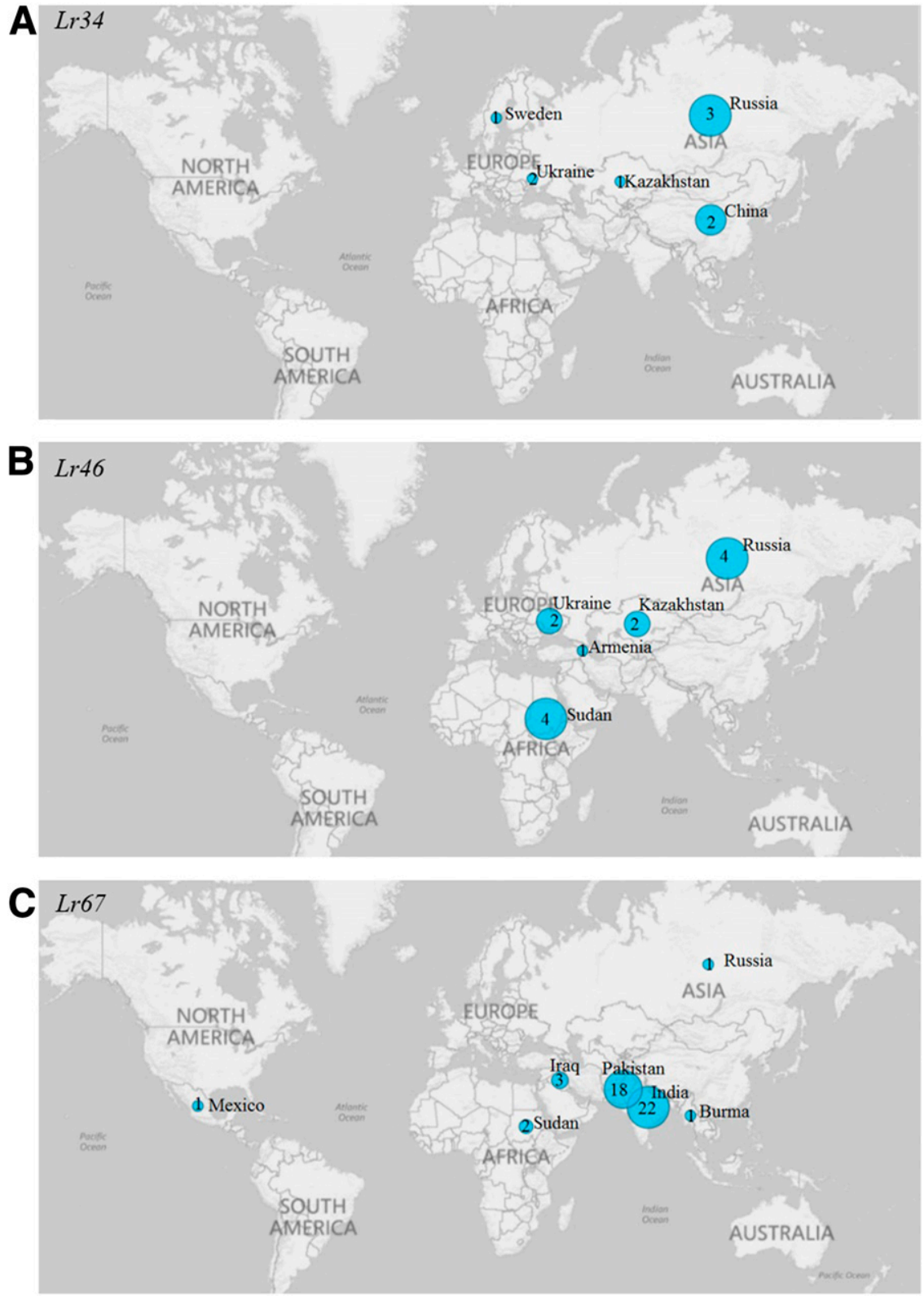

Fig. 1. Geographical distribution of A, Lr34; B, Lr46; and C, Lr67 in the Vavilov wheat diversity panel. Sizes of the circles are proportional to the number of lines carrying alleles for resistance. Lines with gene combinations were tallied individually for the respective gene total. Lines lacking origin information were not displayed. 
$\mathrm{B}$, and $\mathrm{C}$ ). Some lines with unknown origin also carried the APR genes: 3 carried Lr34, 12 carried Lr46, and 3 carried Lr67 (not presented in Figure 1). Two lines carried $L r 34$ and $L r 46$ in combination, while three lines carried $\mathrm{Lr} 46$ and $\mathrm{Lr67}$ in combination. The mean disease response for lines carrying known APR genes ( $L r 34, L r 46$, and $\operatorname{Lr67}$ ) evaluated under AGC was 4.2, 5.9, and 6.1 on the 0-to9 scale, respectively (Fig. 2). Notably, none of the lines carried all three APR genes. $L r 34$ was detected in lines from Russia $(n=3)$, unknown origin $(n=3)$, China $(n=2)$, Ukraine $(n=2)$, Kazakhstan $(n=1)$, and Sweden $(n=1)$ (Fig. 1A). Lr46 was present in lines from Russia $(n=4)$, Sudan $(n=4)$, Kazakhstan $(n=2)$, Ukraine $(n=2)$, Armenia $(n=1)$, and unknown origin ( $n=12$ ) (Fig. 1B). In the case of $L r 67$, the allele for resistance was predominantly observed in lines from India $(n=22)$ and Pakistan $(n=18)$ and, to a lesser extent, it was present in lines from Iraq $(n=3)$, Sudan $(n=2)$, Myanmar $(n=1)$, Russia $(n=1)$, Mexico $(n=1)$, and unknown origin $(n=3)$ (Fig. 1C).

Of the 136 SSD lines classed as landraces, 53 carried known APR genes: $\operatorname{Lr} 34(n=4), \operatorname{Lr} 46(n=7)$, and $\operatorname{Lr} 67(n=42)$. Only one breeding line carried a known APR gene (i.e., $L r 46$ ). However, a number of lines classed as cultivars carried $\operatorname{Lr34}(n=4), \operatorname{Lr} 46(n=7)$, and $\operatorname{Lr67}(n=1)$. None of the five durum lines carried $L r 34, L r 46$, or $\operatorname{Lr} 67$.

Rapid phenotyping: Seedling stage. Of the 300 lines, 73 displayed variable levels of resistance $(<7)$ and 220 displayed susceptibility ( $\geq 7$ ) against pathotype 104-1,2,3,(6),(7),11,13. Seven lines failed to germinate. Among the S lines, 13 scored 7, 77 scored 8, 55 scored 8.5 , and 75 scored 9 (based on a 0-to-9 scale). The majority of the lines deemed seedling susceptible were of unknown origin $(n=75)$, followed by lines originating from India $(n=31)$, Pakistan $(n=24)$, and Russia $(n=29)$. The majority of lines that displayed seedling resistance were from Russia $(n=21)$, Ukraine $(n=6)$, and Pakistan $(n=6)$. Nineteen lines of unknown origin also displayed resistance. Notably, two lines displayed an HR (i.e., IT 0;) and these originated from Pakistan and Tajikistan. The frequency distribution for seedling response was skewed toward susceptibility on the 0-to-9 scale (Fig. 3A). The disease standards such as Thatcher, Avocet, Avocet $+L r 34$, and Avocet $+L r 46$ were found to be seedling susceptible, with an IT ranging from 7 to 9 on the 0 -to- 9 scale.

Rapid phenotyping: Adult stage. In the integrated adult plant experiment under AGC, the 300 lines displayed a complete range of LR response types, ranging from $\mathrm{R}$ to $\mathrm{S}$, when inoculated with pathotype 104-1,2,3,(6),(7),11,13 (Fig. 3B). As described above, seven lines failed to germinate. Therefore, under AGC, 139 lines displayed resistance, while 153 depicted susceptibility. One line was evaluated at the seedling stage but not at the adult stage. The majority of $\mathrm{R}$ lines

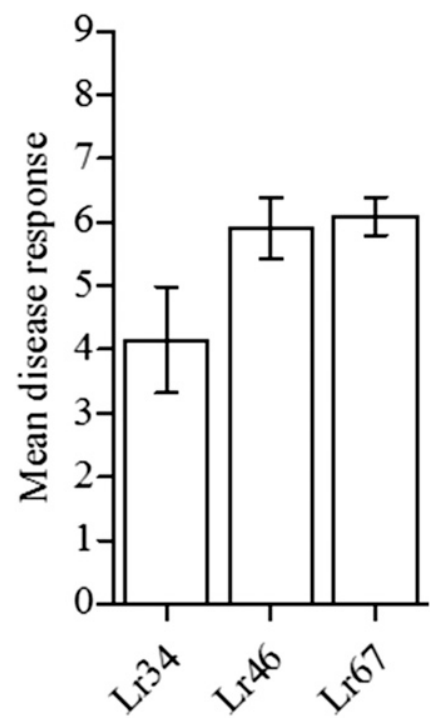

Fig. 2. Mean leaf rust response observed under accelerated growth conditions (flag-2 leaf) for lines carrying known adult plant resistance genes: Lr34 (12 lines), Lr46 (25 lines), and Lr67 (51 lines). Error bars display the standard error of the mean for lines carrying the respective gene. were of unknown origin $(n=43)$, followed by Russia $(n=33)$, India $(n=16)$, and Pakistan $(n=12)$ (Fig. 3B). Likewise, the majority of $\mathrm{S}$ lines were of unknown origin $(n=51)$, followed by India $(n=$ $18)$, and Pakistan $(n=18)$. On the basis of seedling susceptibility and resistance displayed at the adult growth stage, 86 lines were deemed to carry APR, while 54 lines displayed ASR, defined by resistance displayed at both seedling and adult growth stages. The frequency distribution of adult plant response to LR was more evenly distributed in comparison with the seedling response (Fig. 3B).

Thatcher displayed a very susceptible response (VS; IT 9), with urediniospores freely sporulating on leaves. Avocet displayed a resistant-moderately resistant (RMR) response, with IT 4, because Avocet carries race-specific APR gene Lr13, which is effective against the pathotype used for screening under AGC. Avocet $+L r 34$ displayed an RMR response (i.e., IT 4), while Avocet+Lr46 demonstrated a moderately resistant (MR) response (i.e., IT 6).

Identification of novel sources of APR. In total, 86 lines were deemed to carry APR based on the integrated seedling and adult plant phenotyping performed under AGC. Of these, 36 lines carried known APR genes based on results from marker screening. Therefore, the screening process identified 50 wheat lines carrying potentially novel sources of APR to LR.

Field evaluation. In 2014, the 50 lines carrying potentially novel APR were evaluated in the field using the same pathotype (i.e., 104$1,2,3,(6),(7), 11,13)$ used for initial screening of lines at seedling and adult stage under AGC. Of the 50 lines, 29 were considered R, while 21 were considered $\mathrm{S}$ in the field; that is, 2 were moderately susceptible (MS; 6 on 0 -to-9 scale), 3 were moderately susceptible to susceptible (MSS; 7 on 0 -to-9 scale), and 16 were S ( 8 on 0 -to- 9 scale). Despite some variation in response, field phenotypes observed in 2014 corresponded well with those observed under AGC $\left(R^{2}=0.82\right)$.

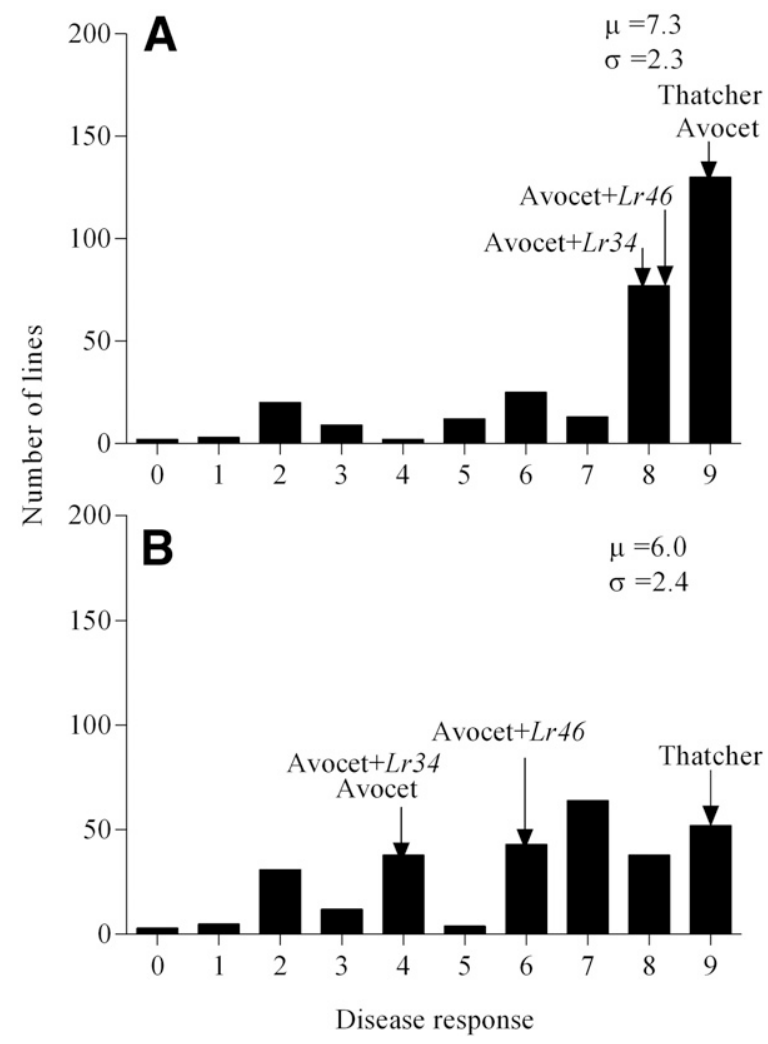

Fig. 3. Frequency distribution of leaf rust response for 300 wheat lines evaluated at $\mathbf{A}$, seedling stage (standard glasshouse) and $\mathbf{B}$, adult stage under accelerated growth conditions (flag-2 leaf). The disease response for seedling and adult stage under accelerated growth conditions was collected using the 0-to-4 Stakman scale and converted to the 0 -to- 9 scale (displayed) 
In 2015, the set of 50 lines were again evaluated in the field but using a mixture of two different pathotypes: 104-1,2,3,(6),(7),11,13 and 76-1,3,5,7,9,10,12,13+Lr37. In 2015, 13 lines displayed resistance and 37 displayed varying levels of susceptibility. In total, 16 lines displayed resistance at the adult stage under AGC and in the field in 2014 but displayed susceptibility in 2015 (Fig. 4). These lines likely carry race-specific APR Lr13 and Lr37, because the pathotype mixture used in 2015 had additional virulence for Lr13 and Lr37. Similarly, the disease standard Avocet, which carries $L r 13$, displayed APR in 2014; however, it displayed a VS response in 2015 (Fig. 5). In contrast, Avocet+Lr34 and Avocet+Lr46 displayed stable resistance even against the Lrl3-virulent pathotype and high inoculum pressure (Fig. 5). Following the 2 years of field evaluation, 13 lines were deemed to carry stable APR and likely harbor novel genes (Fig. 5).

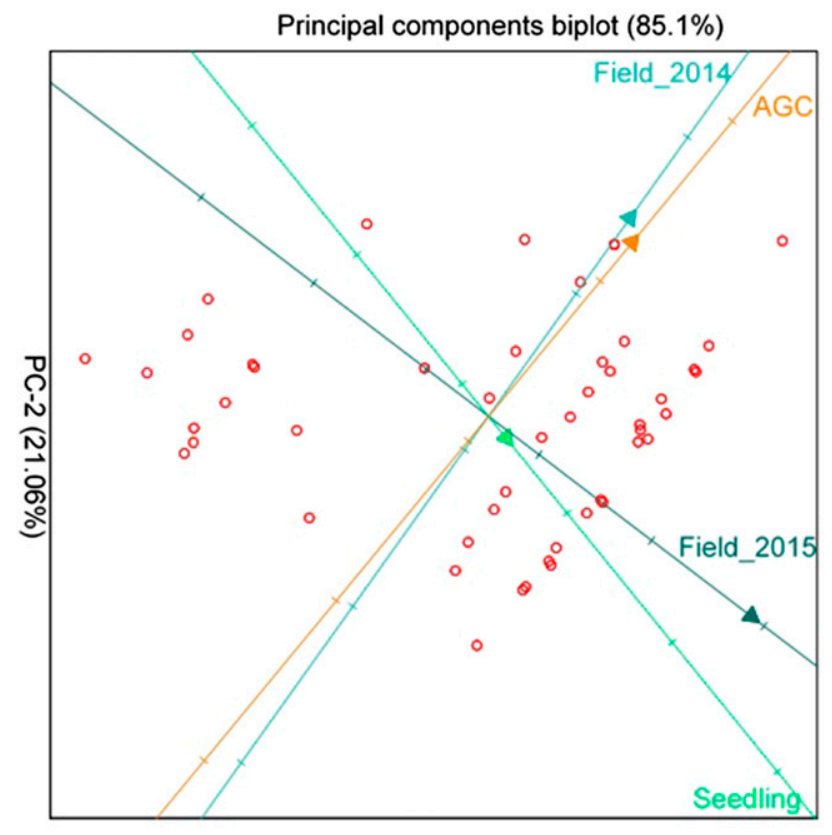

\section{PC-1 (64.04\%)}

Fig. 4. Biplot displaying results from principal component (PC) analysis of leaf rust response for the 50 lines identified following initial screening. Leaf rust response was obtained in the following four experiments: (i) seedling, (ii) adult stage under accelerated growth conditions, (iii) field in 2014, and (iv) field in 2015. Displayed PCs (i.e., PC1 and PC2) account for $85.1 \%$ of the variation.

\section{Discussion}

In this study, we identified useful sources of APR to LR by effectively mining diverse wheat lines from the VIR. We anticipate that this will accelerate the isolation of novel genes, which are required to diversify resistance factors in breeding material.

Of the three known APR genes screened using PCR markers, Lr34 was the least common (i.e., only 12 lines). The gene was mostly observed in lines with unknown origin, followed by lines from China, Kazakhstan, Russia, Ukraine, and Sweden. A previous study by Dakouri et al. (2014) suggests that Lr34 likely originated from Asia, specifically China or Japan. Although some Lr34-carrying lines in this study were from China, the presence of the gene in lines from other countries in Central Asia or Europe could have resulted from early movement of wheat germplasm around the world. One of the key cultivars that increased the spread and utilization of Lr34 was 'Frontana', which was used to first characterize the gene in 1966 (Dyck et al. 1966; Singh 1992).

Based on the $\operatorname{cs} L v 46$ marker, the resistance allele for $L r 46$ was present in 25 of the 300 lines in the diversity panel. These lines were largely of unknown origin, followed by lines from Armenia, Kazakhstan, Russia, and Ukraine. It should be noted that $\operatorname{cs} L v 46$ sometimes provides false positives because it is not diagnostic; thus, it is difficult to infer the likely origin of Lr46. However, based on the Lr46-linked marker, the resistance allele was present in one landrace collected in the 1960s from Sudan and also Russian cultivars and breeding lines from 1952 onward. Notably, Lr46 was first characterized in the International Maize and Wheat Improvement Center (CIMMYT) cv. Pavon 76 (Singh et al. 1998).

Lr67 was present in $17 \%$ of the lines evaluated in this study (i.e., 51 lines). The majority of these lines were from India $(n=22)$ and Pakistan $(n=18)$, followed by Russia, Burma, and Iraq. Most of the lines carrying Lr67 from India and Pakistan were landraces, suggesting that the gene originated from this region. Similar observations of the prevalence of $\mathrm{Lr} 67$ in the Punjab were reported by Forrest et al. (2014) and Moore et al. (2015). The high frequency of Lr67 in this diversity panel might be due to the higher proportion of lines collected from Asia (particularly India and Pakistan), likely a result of multiple expeditions conducted by N. I. Vavilov and A. E. Watkins from 1920 to 1930 , followed by succeeding investigators to date. In this diversity panel, $\operatorname{Lr} 67$ was also found in lines from Sudan and Mexico, most likely a result of the early transfer of genetic material across continents. Lr67 was originally detected and characterized in Pakistani landrace PI250413 (Dyck and Samborski 1979; HerreraFoessel et al. 2012; Hiebert et al. 2010).

The integrated phenotyping performed under AGC identified wheat lines displaying both ASR (i.e., hypersensitive flecking and small uredia with necrosis) and varying levels of APR (i.e., restricted

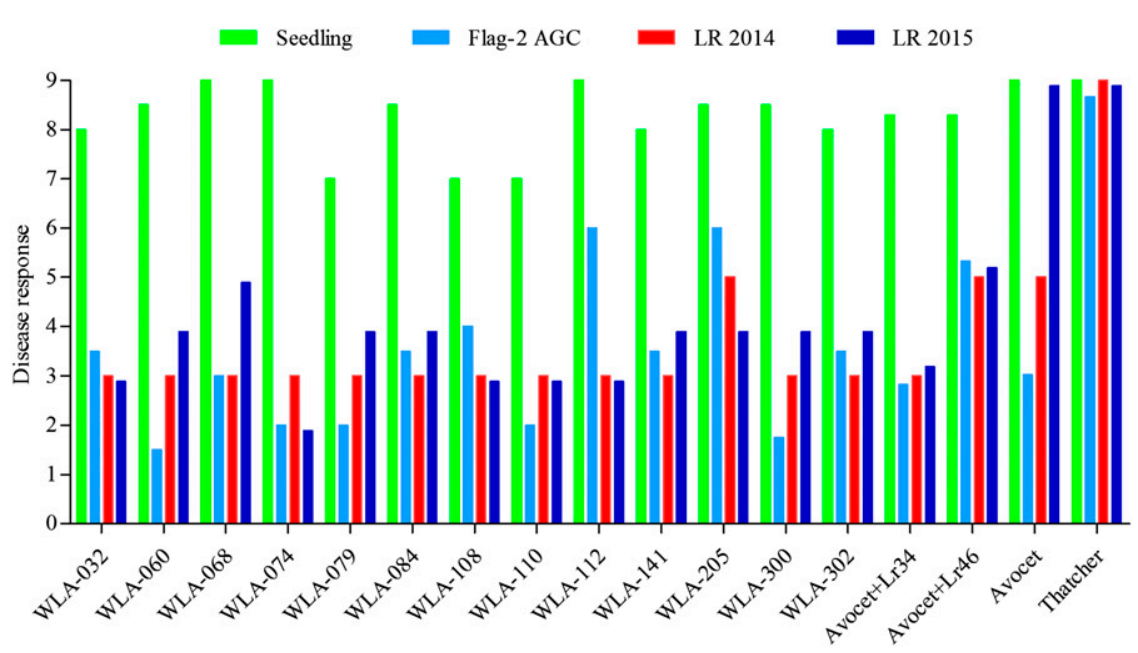

Fig. 5. Comparison of leaf rust $(L R)$ response for the 13 lines carrying novel adult plant resistance, along with disease standards (Thatcher, Avocet, Avocet $L r 34$, and $A v o c e t+L r 46$ ) evaluated at the seedling stage, adult stage (i.e., flag-2 leaf) under accelerated growth conditions (AGC), and in the field in 2014 and 2015. 
sporulation and chlorosis) to $P$. triticina. Following the elimination of lines that carried known APR genes, 50 lines were deemed to carry potentially new sources of APR. Of these, 37 displayed varying levels of susceptibility in at least one of the two field environments. Notably, 16 of the 37 lines appeared to carry race-specific APR gene Lr13 and Lr37 based on their increased susceptibility to the pathotype mix used in 2015 field screening. Other factors contributing to the variability in disease response across adult assays were likely differences in environmental conditions (e.g., temperature, light, and humidity) and growth stage at assessment, which are known to influence expression of APR (Herrera-Foessel et al. 2012; Hickey et al. 2012; Hiebert et al. 2010; Kaul and Shaner 1989; Singh and Huerta-Espino 2003). Cooler temperatures are known to enhance the effectiveness of Lr34; for instance, 13 to $18^{\circ} \mathrm{C}$ compared with $23^{\circ} \mathrm{C}$ (Singh and Huerta-Espino 2003). In this study, the rapid phenotyping assay was performed at controlled temperatures of 17 and $22^{\circ} \mathrm{C}$ (night and day). Under these conditions, lines carrying $L r 34$ displayed higher levels of resistance at the adult plant stage in comparison with lines carrying $L r 46$ or $L r 67$, which is in agreement with previous field studies (Ellis et al. 2014; Hiebert et al. 2010). The assessment under AGC used a single controlled inoculation, whereas assessment in the field was subject to variable weather conditions (e.g., fluctuating temperatures), plant growth stage, and polycyclic pathogen infection (Niks et al. 2015). Therefore, some resistance factors such as a long latent period or small pustule size may be phenotyped more precisely using a single controlled inoculation. APR genes often provide weak or low levels of resistance to LR and thus are often scored as MS or MSS in the field, such as APR gene Lr67 (Hiebert et al. 2010). In this study, we applied strict criteria for resistance (i.e., $\leq$ MRMS). Thus, it is possible that additional lines displaying low susceptibility scores in the field (i.e., MS or MSS) could carry weak APR. These factors might be useful under lower disease pressure or coupled with additional APR.

PCR marker screening and APR phenotypes observed under AGC initially identified 50 lines of interest. Of these, 13 lines consistently displayed moderate to high levels of resistance in the field using a mixture of pathotypes. Interestingly, these lines originated from different geographical regions of the world, including Russia (six), unknown origin (two), India (two), China (one), Chile (one), and Portugal (one). Therefore, they likely carry different sources of resistance. Furthermore, among the 13 lines, there was also diversity in terms of cultivation status: 5 cultivars, 5 landraces, 1 breeding line, and 2 with unknown cultivation status. In addition to the APR genes screened in this study (i.e., $\operatorname{Lr} 13, \operatorname{Lr} 34, \operatorname{Lr} 37, \operatorname{Lr} 46$, and $\operatorname{Lr} 67$ ), a number of other race-specific APR have been cataloged, including $L r 12$, $L r 22$ (alleles a and b), $L r 35, L r 48$, and $L r 49$, plus $L r 68$, which is a non race-specific APR (Ellis et al. 2014; Li et al. 2014; McIntosh et al. 1995). Therefore, to determine whether the genes are novel, fine mapping is required and, if positioned on the same chromosome as these previously cataloged genes, allele testing is needed.

This study highlights the value of historical germplasm to provide the much-needed genetic diversity to improve wheat productivity in the face of climate change and rapidly evolving pathogens. Although only a small selection of 300 wheat lines from VIR were screened, around the world there are hundreds of thousands of viable wheat accessions preserved in seed banks, such as the Svalbard Global Seed Vault and collections based at CIMMYT, the United States Department of Agriculture, and John Innes Centre.

The rapid phenotyping methodology performed under controlled conditions presents a number of advantages: (i) it only requires 7 weeks to complete, (ii) environmental factors are controlled, and (iii) it can be performed all year round. On the other hand, conventional field screening is time consuming (i.e., 4 to 5 months), subject to weather conditions, and can only be performed once a year. Additional accessions in seed banks can be rapidly screened using this approach, which reduces the number for field-based evaluation of resistance. Further, it could be integrated with Focused Identification of Germplasm Strategy or other trait-mining techniques (Mackay et al. 2016).

The sources of resistance identified in this study will be used to generate biparental populations for gene mapping and identification of linked DNA markers, which will assist gene pyramiding. Transfer of resistances could be accelerated by selecting for APR to LR in parallel with rapid generation advance under "speed breeding" or AGC (Mackay et al. 2016).

\section{Acknowledgments}

This research was supported by an Early Career Research Grant and a Ph.D. scholarship from The University of Queensland, Australia. We thank Greg Platz at the Department of Agriculture and Fisheries, Queensland, Australia for providing advice for establishing disease screening nurseries.

\section{Literature Cited}

Asseng, S., Ewert, F., Martre, P., Rötter, R. P., Lobell, D. B., Cammarano, D., Kimball, B. A., Ottman, M. J., Wall, G. W., White, J. W., Reynolds, M. P., Alderman, P. D., Prasad, P. V. V., Aggarwal, P. K., Anothai, J., Basso, B., Biernath, C., Challinor, A. J., De Sanctis, G., Doltra, J., Fereres, E., GarciaVila, M., Gayler, S., Hoogenboom, G., Hunt, L. A., Izaurralde, R. C., Jabloun, M., Jones, C. D., Kersebaum, K. C., Koehler, A.-K., Müller, C., Naresh Kumar, S., Nendel, C., O’Leary, G., Olesen, J. E., Palosuo, T., Priesack, E., Eyshi Rezaei, E., Ruane, A. C., Semenov, M. A., Shcherbak, I., Stöckle, C., Stratonovitch, P., Streck, T., Supit, I., Tao, F., Thorburn, P. J., Waha, K., Wang, E., Wallach, D., Wolf, J., Zhao, Z., and Zhu, Y. 2015. Rising temperatures reduce global wheat production. Nat. Clim. Change 5: 143-147.

Bansal, U. K., Hayden, M. J., Venkata, B. P., Khanna, R., Saini, R. G., and Bariana, H. S. 2008. Genetic mapping of adult plant leaf rust resistance genes Lr48 and Lr49 in common wheat. Theor. Appl. Genet. 117:307-312.

Bariana, H., Miah, H., Brown, G., Willey, N., and Lehmensiek, A. 2007. Molecular mapping of durable rust resistance in wheat and its implication in breeding. Pages 723-728 in: Wheat Production in Stressed Environments. H. T. Buck, J. E. Nisi, and N. Salomón, eds. Springer, Dordrecht, The Netherlands.

Caldwell, R. M. 1968. Breeding for general and/or specific plant disease resistance. Pages 263-272 in: Proc. Third Int. Wheat Genet. Symp. Australian Academy of Sciences, Canberra, Australia.

Chaves, M. S., Martinelli, J. A., Wesp-Guterres, C., Graichen, F. A. S., Brammer, S. P., Scagliusi, S. M., da Silva, P. R., Wiethölter, P., Torres, G. A. M., Lau, E. Y., Consoli, L., and Chaves, A. L. S. 2013. The importance for food security of maintaining rust resistance in wheat. Food Secur. 5:157-176.

Dakouri, A., McCallum, B. D., and Cloutier, S. 2014. Haplotype diversity and evolutionary history of the Lr34 locus of wheat. Mol. Breed. 33:639-655.

Dyck, P., and Samborski, D. 1979. Adult-plant leaf rust resistance in PI 250413, an introduction of common wheat. Can. J. Plant Sci. 59:329-332.

Dyck, P., Samborski, D., and Anderson, R. 1966. Inheritance of adult-plant leaf rust resistance derived from the common wheat varieties Exchange and Frontana. Can. J. Genet. Cytol. 8:665-671.

Ellis, J. G., Lagudah, E. S., Spielmeyer, W., and Dodds, P. N. 2014. The past, present and future of breeding rust resistant wheat. Front. Plant Sci. 5:641.

Forrest, K., Pujol, V., Bulli, P., Pumphrey, M., Wellings, C., Herrera-Foessel, S., Huerta-Espino, J., Singh, R., Lagudah, E., Hayden, M., and Spielmeyer, W. 2014. Development of a SNP marker assay for the Lr67 gene of wheat using a genotyping by sequencing approach. Mol. Breed. 34:2109-2118.

Herrera-Foessel, S. A., Singh, R. P., Huerta-Espino, J., Rosewarne, G. M. Periyannan, S. K., Viccars, L., Calvo-Salazar, V., Lan, C., and Lagudah, E. S. 2012. Lr68: A new gene conferring slow rusting resistance to leaf rust in wheat. Theor. Appl. Genet. 124:1475-1486.

Hickey, L. T., Wilkinson, P. M., Knight, C. R., Godwin, I. D., Kravchuk, O. Y., Aitken, E. A., Bansal, U. K., Bariana, H. S., De Lacy, I. H., and Dieters, M. J. 2012. Rapid phenotyping for adult-plant resistance to stripe rust in wheat. Plant Breed. 131:54-61.

Hiebert, C. W., Thomas, J. B., McCallum, B. D., Gavin Humphreys, D., DePauw, R. M., and Hayden, M. J. 2010. An introgression on wheat chromosome 4DL in RL6077 (Thatcher*6/PI 250413) confers adult plant resistance to stripe rust and leaf rust (Lr67). Theor. Appl. Genet. 121:1083-1091.

Huerta-Espino, J., Singh, R. P., Germán, S., McCallum, B. D., Park, R. F., Chen, W. Q., Bhardwaj, S. C., and Goyeau, H. 2011. Global status of wheat leaf rust caused by Puccinia triticina. Euphytica 179:143-160.

Jørgensen, L. N., Hovmøller, M. S., Hansen, J. G., Lassen, P., Clark, B., Bayles, R., Rodemann, B., Flath, K., Jahn, M., Goral, T., Jerzy Czembor, J., Cheyron, P., Maumene, C., De Pope, C., Ban, R., Nielsen, G. C., and Berg, G. 2014. IPM strategies and their dilemmas including an introduction to www.eurowheat.org. J. Integr. Agric. 13:265-281.

Kaul, K., and Shaner, G. 1989. Effect of temperature on adult-plant resistance to leaf rust in wheat. Phytopathology 79:391-394.

Krattinger, S. G., Lagudah, E. S., Spielmeyer, W., Singh, R. P., Huerta-Espino, J., McFadden, H., Bossolini, E., Selter, L. L., and Keller, B. 2009. A putative ABC transporter confers durable resistance to multiple fungal pathogens in wheat. Science 323:1360-1363.

Lagudah, E. S. 2011. Molecular genetics of race non-specific rust resistance in wheat. Euphytica 179:81-91.

Lagudah, E. S., Krattinger, S. G., Herrera-Foessel, S., Singh, R. P., Huerta-Espino, J., Spielmeyer, W., Brown-Guedira, G., Selter, L. L., and Keller, B. 2009. 
Gene-specific markers for the wheat gene $\operatorname{Lr} 34 / \mathrm{Yr} 18 / \mathrm{Pm} 38$ which confers resistance to multiple fungal pathogens. Theor. Appl. Genet. 119:889-898.

Li, Z., Lan, C., He, Z., Singh, R. P., Rosewarne, G. M., Chen, X., and Xia, X. 2014. Overview and application of QTL for adult plant resistance to leaf rust and powdery mildew in wheat. Crop Sci. 54:1907-1925.

Loegering, W. 1959. Methods for Recording Cereal Rust Data. USDA International Spring Wheat Nursery.

Mackay, M. C., Street, K. A., and Hickey, L T. 2016. Toward more effective discovery and deployment of novel plant genetic variation: reflection and future directions. Pages 139-150 in: Applied Mathematics and Omics to Assess Crop Genetic Resources for Climate Change Adaptive Traits. A. Bari, A. B. Damania, M. Mackay, and S. Dayanandan, eds. CRC Press, Boca Raton, FL.

McIntosh, R. A., Wellings, C. R., and Park, R. F. 1995. Wheat Rusts: An Atlas of Resistance Genes. Csiro Publishing, East Melbourne, Australia.

Mitrofanova, O. P. 2012. Wheat genetic resources in Russia: Current status and prebreeding studies. Russ. J. Genet. Appl. Res. 2:277-285.

Moore, J. W., Herrera-Foessel, S., Lan, C., Schnippenkoetter, W., Ayliffe, M., Huerta-Espino, J., Lillemo, M., Viccars, L., Milne, R., Periyannan, S., Kong, X., Spielmeyer, W., Talbot, M., Bariana, H., Patrick, J. W., Dodds, P., Singh, R., and Lagudah, E. 2015. A recently evolved hexose transporter variant confers resistance to multiple pathogens in wheat. Nat. Genet. 47:1494-1498.

Niks, R. E., Qi, X., and Marcel, T. C. 2015. Quantitative resistance to biotrophic filamentous plant pathogens: Concepts, misconceptions, and mechanisms. Annu. Rev. Phytopathol. 53:445-470.

Park, R. F., and Bariana, H. S. 2013. First detection of wheat leaf rust pathotype 76-1,3,5,7,9,10,12+Lr37 in Western Australia. Cereal Rust Rep. 11:1-4.

Park, R. F., Bariana, H. S., Wellings, C. R., and Wallwork, H. 2002. Detection and occurrence of a new pathotype of Puccinia triticina with virulence for $L r 24$ in Australia. Crop Pasture Sci. 53:1069-1076.

Park, R. F., Singh, D., and William, C. 2015. Rust pathotype update, August 2015. Cereal Rust Rep. 13:1-2.
Park, R. F., and Wellings, C. R. 2011. Cereal rust survey 2011 progress report: New leaf rust pathotype and re-emerging stripe rust pathotype. Cereal Rust Rep. 9:1-3.

Peterson, R. F., Campbell, A., and Hannah, A. 1948. A diagrammatic scale for estimating rust intensity on leaves and stems of cereals. Can. J. Res. 26c:496-500.

Ray, D. K., Mueller, N. D., West, P. C., and Foley, J. A. 2013. Yield trends are insufficient to double global crop production by 2050. PLoS One 8:e66428.

Riaz, A., Hathorn, A., Dinglasan, E., Ziems, L., Richard, C., Singh, D. Mitrofanova, O., Afanasenko, O., Aitken, E., Godwin, I., and Hickey, L. Into the vault of the Vavilov wheats: Old diversity for new alleles. Genet. Resour. Crop Evol. In press. doi:10.1007/s10722-016-0380-5

Riaz, A., Periyannan, S., Aitken, E., and Hickey, L. 2016. A rapid phenotyping method for adult plant resistance to leaf rust in wheat. Plant Methods 12:17.

Singh, R., and Huerta-Espino, J. 2003. Effect of leaf rust resistance gene Lr34 on components of slow rusting at seven growth stages in wheat. Euphytica 129: 371-376.

Singh, R., Mujeeb-Kazi, A., and Huerta-Espino, J. 1998. Lr46: A gene conferring slow-rusting resistance to leaf rust in wheat. Phytopathology 88:890-894.

Singh, R. P. 1992. Genetic association of leaf rust resistance gene $L r 34$ with adultplant resistance to stripe rust in bread wheat. Phytopathology 82:835-838.

Singh, R. P., Herrera-Foessel, S., Huerta-Espino, J., Singh, S., Bhavani, S., Lan, C., and Basnet, B. R. 2014. Progress towards genetics and breeding for minor genes based resistance to Ug99 and other rusts in CIMMYT highyielding spring wheat. J. Integr. Agric. 13:255-261.

Spielmeyer, W., Mago, R., Wellings, C., and Ayliffe, M. 2013. Lr67 and Lr34 rust resistance genes have much in common-they confer broad spectrum resistance to multiple pathogens in wheat. BMC Plant Biol. 13:96.

Stakman, E., Stewart, D., and Loegering, W. 1962. Identification of physiologic races of Puccinia graminis var. tritici. United States Department of Agriculture, Washington, DC.

UN. 2015. World Population Prospects: The 2015 Revision World Population 2015 Wallchart. ST/ESA/SER.A/378 